\title{
A developmental investigation of selective attention to graphic, phonetic, and semantic information in words
}

\author{
SANDRA M. CONDRY, MARGARET McMAHON-RIDEOUT, and ANITA A. LEVY \\ Cornell University, Ithaca, New York 14853
}

\begin{abstract}
Second and fifth-grade and college-age subjects made similarity judgments on sets of three words that required attention to orthographic, phonetic, or semantic information. Accuracy and speed increased with age. Even the youngest subjects were able to perform the task of selecting a given feature of a word reasonably well. Differences in difficulty among the three tasks decreased with age, suggesting a developmental change (primarily between second and fifth grade) toward facility in extracting phonetic and semantic information from words. The presence of confusable, potentially relevant information had detrimental effects overall which decreased with age and varied with the type of task and type of distractor. For all ages, performance was better when all trials of a particular task were blocked together than when trials of the three tasks were randomly ordered.
\end{abstract}

Printed words offer several types of information: semantic and morphological, syntactic, graphic and orthographic, and phonetic. Gibson (1971) has called these types feature classes. When reading, one's purpose determines which class of information is most relevant. Although we generally read for meaning, we may focus on orthography when correcting papers or looking up a name in the phone book, and on sound when reading poetry. Performing any of these tasks requires the ability to extract and work with one class of information in preference to another.

The present study is concerned with the development of selective attention to particular classes of information offered by printed words. It has been established that 7-year-olds can attend selectively to graphic distinctive features in a search task, using strategies similar to those of adults (Gibson \& Yonas, 1966), and that they can perform some types of phonemic analysis (Golden, Note 1). The present study provides a more complete test of readers' abilities to work with specific classes of information. Children of two grade levels and college students

An earlier version of this report was presented at the annual meeting of the Eastern Psychological Association, New York, April 1976. The authors wish to thank Dr. Eleanor J. Gibson for her extensive help in devising and writing up this study. Dr. Ulric Neisser's critical reading of an earlier draft was very helpful. We are appreciative of the cooperation extended to us by the principal of Northeast Elementary School, Michael Ouckama, and by the teachers and students. $O$. Brian Allen and Donald Schuirmann provided invaluable assistance in mapping out the statistical analyses. This research was supported by grants from the National Institute of Child Health and Human Development (Grant HDOO381-05) and the National Institute of Mental Health (Grant MHO8520-12). Requests for reprints may be addressed to Sandra Condry, Psychology Department, Cornell University, Ithaca, New York 14853. made similarity judgments of features from three classes-graphic (which of two words looks like a target word), phonetic (which of two words rhymes with a target word), and semantic (which of two words is synonymous with a target word). Response latencies were recorded. Developmental changes in ease of extracting each type of information were assessed by testing for interactions between grade level and feature class. It was expected that adults would have little difficulty with any of the tasks, while second graders would have more difficulty extracting semantic and phonetic than graphic information.

Selective attention presumably entails ignoring some information. There is considerable evidence to support the hypothesis that young children are more susceptible than older children and adults to distraction by task-irrelevant information (Clifton \& Bogartz, 1968; Maccoby \& Konrad, 1966, 1967; Pick, Christy, \& Frankel, 1972; Smith, Kemler, \& Aronfreed, 1975; Strutt, Anderson, \& Well, 1975).

The present experiment explored, developmentally, the ability to ignore irrelevant information in the word-judgment task. On each trial, the subject had to decide which one of the two choice words was more similar to the target in the specified way. The relationship of the other word (distractor) to the target was manipulated so that the distracting influence of irrelevant semantic, graphic, or phonetic similarity could be assessed by comparison with the effects of control distractors. Control distractors were words that bore no particular relation to the target. It was hypothesized that the younger children, who had had less experience in working with these classes of information, would have more difficulty attending to them selectively. For instance, when 
searching for a word that rhymed with the target, it was expected that a young child would be distracted by a word that looked like the target. (In this study, rhyming words were not graphically similar, and graphically similar words did not rhyme.) If the distractor was dissimilar to the target, confusion should not occur. We expected that trials with control distractors would be easier for young children than trials with feature distractors (i.e., distractors similar to the target along an irrelevant dimension).

The pattern of distractor effects on particular tasks at each age is also of interest for the light it sheds on reading processes. It is possible that at one level of reading expertise, some classes of information are less clearly differentiated than they are at an earlier or later level of reading ability. For example, it is often hypothesized that young readers process the pronunciation of each word before processing its meaning, and that progress in reading entails learning to bypass the sound of a word. On this hypothesis, we would expect the effect of the rhymes distractor on the means task to decrease with age, since younger readers are using the sound of a word while older readers aren't. The second-grade data provide evidence on this point. If, on the means task, the rhymes distractor was no more disruptive of performance than the control distractor, it would indicate that second graders were accessing the meaning without first decoding the sound.

Besides the ability to attend to relevant and ignore irrelevant information, adaptable reading requires flexibility in applying reading skills. A study of flexibility in reading for comprehension found differences between good and poor readers of college age (Anderson, 1937). Developmental studies using nonreading tasks have found that older children are more flexible in switching strategies than are younger children (Doyle, 1973; Pick \& Frankel, 1973). In the present study, the three tasks-looks, rhymes, and means-were presented to half of the subjects in blocks and to the other half in a random order from trial to trial. It was hypothesized that frequent changes in the task would be more disruptive to the younger children's performance than to that of older children and adults.
In summary, while older subjects were expected to have faster overall reaction times, grade level was expected to interact with (a) the specific type of information to be extracted, (b) the presence of distractors that resembled the standard word in irrelevant ways, and (c) with blocking of trials.

\section{METHOD}

\section{Design}

Independent variables were grade, blocking, or randomization of the tasks in presentation, task, and distractor. The design was a 3 by 2 by 3 by 4 factorial with three grade levels (second, fifth, and college) and two groupings of the tasks (blocked and random) between subjects. The three tasks (looks, rhymes, and means) and the four distractor treatments (control, looks, rhymes, means) were within-subject variables. Since a task and a distractor of the same type could not be presented together, the graphicgraphic, phonetic-phonetic, and semantic-semantic combinations of task and distractor were not used. The task and distractor treatments were thus incompletely crossed, yielding nine Task by Distractor combinations.

\section{Subjects}

The children were 42 second graders (16 boys, 26 girls) ${ }^{1}$ and 50 fifth graders ( 30 boys, 20 girls) attending school in a middle class neighborhood in Ithaca, New York. The adults were 20 students (10 male, 10 female) attending Cornell University. Within each grade, the subjects were randomly assigned to the two groups for task presentation.

\section{Materials}

On each trial of the experiment, the subject responded to a three-word item. Examples of the nine types of items are shown in Table $1 .^{2}$ Each item consisted of three words: a target, a correct answer, and an incorrect distractor. Each word appeared once in the set of items. The words were selected for likely familiarity to young children. Most were monosyllabic, and all had frequency ratings of A or AA in the Thorndike-Lorge (1944) count.

On 30 trials, the correct answer "looked like" the target words; this was the looks task. Look-alike words had the same number of letters and differed only in one letter (e.g., roam and room). They did not rhyme and were not synonymous. In another 30 trials, the correct answers rhymed with the target words (rhymes task). Rhymes had the same pronunciation on their ending vowel and consonant sounds (e.g., ear and here). In the remaining 30 trials (means task), the correct answers were synonyms of the targets (e.g., boat and ship), but neither looked like nor rhymed with them.

The incorrect distractor words were of four types: looks, rhymes, means, and control. A control distractor bore no obvious relation to the target word. A looks distractor looked like, a

Table 1

Examples of the Nine Task by Distractor Conditions

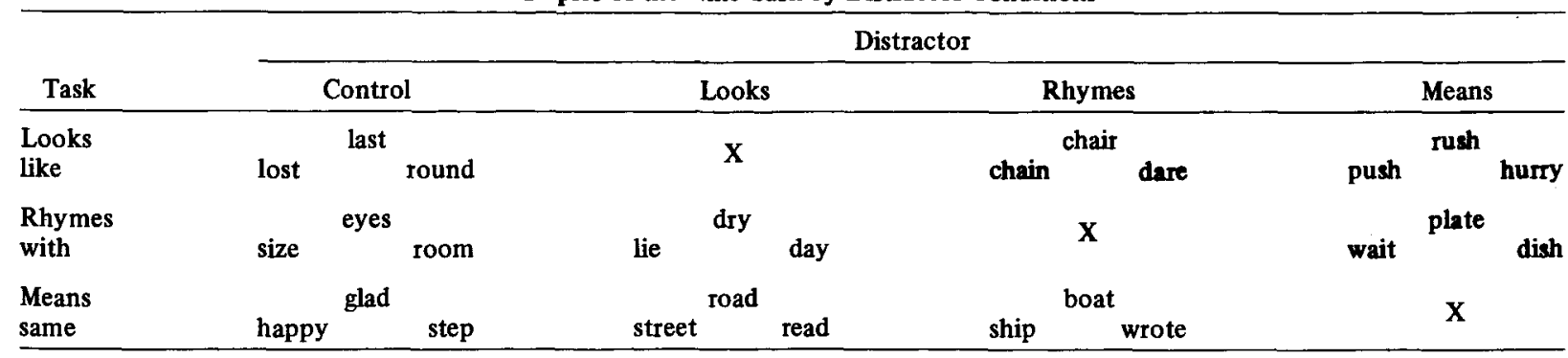


rhymes distractor rhymed with, and a means distractor meant the same as the target by the criteria described above. The $\mathbf{3 0}$ sets of words used with each task included 10 with control distractors and 10 with each of the two distractor types that were irrelevant to that task.

The three words comprising each item were typed (in plain, lowercase letters) on a white card in pyramidal arrangement, with the target above and the correct answer and the distractor at equal distances below to the left or right. The correct answer appeared in the right-hand position in half of the items, and in the left on the rest. The cards were photographed to make blackand-white slides.

Two basic arrangements of item presentation were usedblocked and random. In the blocked grouping, the 30 items for a task were presented one after another, followed by 30 for the second task, and 30 for the third. The items were randomly ordered within a task block, and each subject received one of six orders of the three blocks.

In the random grouping, the 90 slides were arranged in a random sequence and subjects were randomly assigned to a backward or forward presentation of that sequence.

The experimental materials also included 10 practice slides, constructed in the same manner as the test slides, and three demonstration slides, each showing two look-alike, rhyming, or synonymous word pairs.

\section{Apparatus and Procedure}

Each subject was tested individually in a laboratory trailer on the school grounds, or in the laboratory at the university. The subject was seated at a table facing a $45 \times 45 \mathrm{~cm}$ translucent screen. Before him on the table was a response box with two large buttons-one red, one green. The subject was instructed as follows: "I want you to play a word game with me. When you read you probably notice that some words sound alike-they make rhymes. [The experimenter presented a slide showing two pairs of rhyming words, and read them aloud.] Some words look alike-they have the same number of letters and many of the letters are the same. [The experimenter presented a slide showing two pairs of look-alike words.] Some words mean about the same thing--they are synonyms. When you're talking you can use either word and it doesn't make much difference. [The experimenter presented a slide showing two pairs of synonyms, and read them aloud.]" A looks-task practice slide was then presented and the subject was asked to point to the word which looked like the top (target) word. When the subject responded correctly, he was told that he should answer by pushing the button which was on the same side as the correct word-" "the green button if the word on this side is the answer and the red button if the word on that side is the answer." The experimenter demonstrated if the subject did not understand.

After the subject had practiced pushing the correct button for a rhymes-task and a means-task slide, he was told that he would see a number of slides in a row (uninterrupted), that the experimenter would tell him which kind of word to look for ("looks like," "rhymes with," "means the same"), and that he should watch the screen for the top (target) word, listen for the experimenter's announcement, decide on the answer, and push the correct button as quickly as possible, and then prepare for the next trial.

The experimenter proceeded to present 7 more practice slides and the 90 test slides, interrupting only to correct errors made on the practice trials or to remind the subject to pay close attention.

The slides were back-projected by a Carousel projector. At the beginning of a trial, the slide was in place, but was not projected until the experimenter released a separate shutter which activated a Hunter digital timer. When the subject pressed either of the two buttons on the response board, the shutter closed, the timer halted, and a counter in front of the experimenter indicated which button had been pressed. The experimenter (partially hidden from the subject's view by the screen) recorded which button had been pressed and the RT in hundredths of a second. The timer and counter were then reset and the Carousel advanced to the next slide. Each trial began with the simultaneous announcement of the task and opening of the shutter. The time between trials thus amounted to about $3 \mathrm{sec}$. Each trial was immediately preceded by the spoken signal "ready."

\section{RESULTS}

The data were reduced by obtaining, for each subject, the median latency of the correct responses made to the 10 items representing each combination of task and distractor. These median scores (nine for each subject) were the basis of all further analyses.

The analysis of variance was adapted for unequal ns (in Grade by Grouping cells) and for the incompletely crossed factors (three empty Task by Distractor cells) by using the multiple regression technique to obtain sequential sums of squares. A sum of squares thus obtained reflected the variance attributable to a given factor (or interaction) after the variance associated with factors not orthogonal to it had been removed. The appropriate degrees of freedom for $F$ tests were determined by a procedure suggested by Winer $(1971$, p. 523).

All four of the main effects (grade, grouping of trials, nature of task, type of distractor) were found to be statistically significant. Table 2 presents the average RTs for blocked and random groupings at each grade level. Reaction times of older subjects were, overall, more rapid than those of younger subjects $[F(2,106)=72.4, p<.01]$. The main effect of grouping was also significant: shorter RTs resulted when items of a task were presented in a block than when items of the three tasks were interspersed $[F(1,106)=14.7, p<.01]$. The blocked arrangement, relative to the random, was somewhat more facilitating for the second graders than it was for fifth graders and adults, but the interaction (Grade by Grouping) was not significant $[F(2,106)=.92$, $p>.25]$. The simple effect of grouping was significant for adults $[F(1,18)=11.57, p<.01]$, for fifth graders $[F(1,48)=7.12, p<.025]$, and for second graders $[F(1,40)=6.08, p<.025]$.

There were no significant interactions between grouping and other main effects. For the three age

Table 2

Grouping of Tasks

\begin{tabular}{lcc}
\hline Grade & Blocked & Unsystematic \\
\hline 2 & 267 & 339 \\
5 & 165 & 204 \\
Adult & 101 & 125 \\
\hline
\end{tabular}

Note-Values given are average reaction times in centiseconds for blocked and unsystematic grouping of the tasks at each grade level. 
groups combined, blocking the trials of a task did not affect the three tasks differentially, i.e., the Task by Grouping interaction was not significant $[F(2,313)=.67, p>.25]$. The effects of grouping on task, furthermore, did not differ for the three grade levels; the Grade by Task by Grouping interaction was nonsignificant $[F(2,260)=.33, p>.25]$. The effect of distractors was likewise unaffected by the blocking of trials. Over all grades, the Grouping by Distractor interaction was nonsignificant $[F(3,314)$ $=1.16, \mathrm{p}>.25]$. The Grade by Grouping by Distractor interaction was also nonsignificant $[F(3,261)$ $=.93, p>.25]$. Blocking the trials of a task thus produced a uniform facilitation of response time, independent of the type of task or distractor, and (except for the very slight Grade by Grouping interaction) of grade level. Because grouping did not interact with other variables, the data from blocked and random conditions were pooled; the effects reported below are based on the combined data from groups of subjects receiving blocked and random trials.

The effect of task on the three grade levels is illustrated in Figure 1, which shows the mean RTs for the three tasks in the control distractor condition. The significant main effect of task $[F(2,313)=146.3$, $\mathrm{p}<.01$ ] cannot be taken at face value because the different tasks were accompanied by different types of distractors. Similar looking distractors, for instance, occurred only on the rhymes and means task, not on the looks task. Therefore, a separate analysis was performed on the 10 trials in each task that used only control distractors. The task effect considered with only control distractors, and collapsed over grade and grouping, was significant $[F(2,212)$

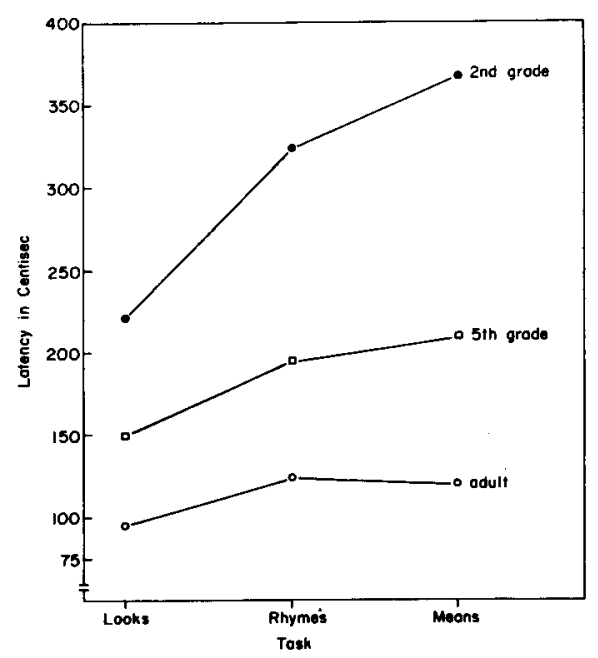

Figure 1. Mean RT of second-grade, fifth-grade, and adult subjects on trials with control distractors for each of the three tasks.

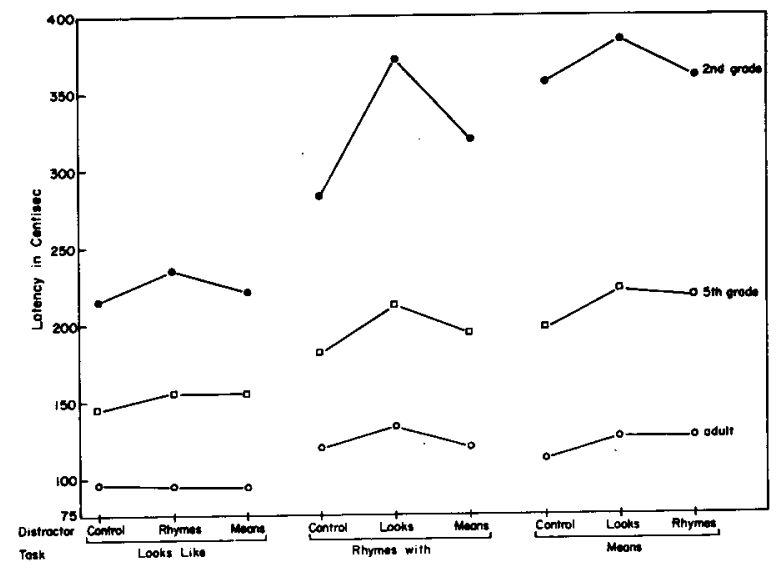

Figure 2. Mean RT of second-grade, fifth-grade, and adult subjects on the nine task-distractor conditions.

$=59.4, \mathrm{p}<.01]$. In both analyses, the looks task had the shortest RTs and the means task the longest. Both analyses also yielded a significant Grade by Task interaction [overall: $F(4,313)=20.8, p<.01$; control distractors only: $F(4,212)=12.3, \mathrm{p}<.01$ ].

The Grade by Task interaction was analyzed by planned contrasts of task differences between the two younger grades. Each contrast compared the Grade 2 difference in the average RT on two tasks with the Grade 5 difference in average RT between the same two tasks. The tests showed that the rhymes task compared to looks task was more difficult for second graders than for fifth graders $[F(1,260)=30.6, p<$ .011 , as was the means task compared to the looks task $[F(1,260)=66.6, p<.01]$. Likewise, the means task compared to the rhymes task was more difficult for second than for fifth graders $[F(1,260)=6.6$, $\mathrm{p}<.05]$. At all ages, the looks task was easiest. The tendency to respond more slowly to the means and rhymes tasks was most pronounced among second graders and decreased with age. For adults, the means and rhymes tasks were equally difficult. ${ }^{3}$

Since the main effect of distractor $[F(3,313)=$ $16.2, p<.01]$ was confounded with that of task, a separate test of distractor effectiveness was required. The test performed was a comparison between the effects of the looks, rhymes, and means distractors combined, and the effect of the control distractor. ${ }^{4}$ This test was significant $[F(1,313)=29.4, p<.01]$, indicating that the three feature distractors were interfering relative to the control distractor, when compared over grade, grouping, and task.

The simple effect of distractors at each grade level was assessed by the same test. The control distractor vs. other distractors comparison was significant at each grade level $(p<.01)$. Much of the distractor effect on all grades was created by the large effect of the looks distractor, as can be seen in Figure 2, 
Table 3

Effects of Feature Distractors Compared with Control Distractors for Three Tasks at Three Grade Levels, Based on RT

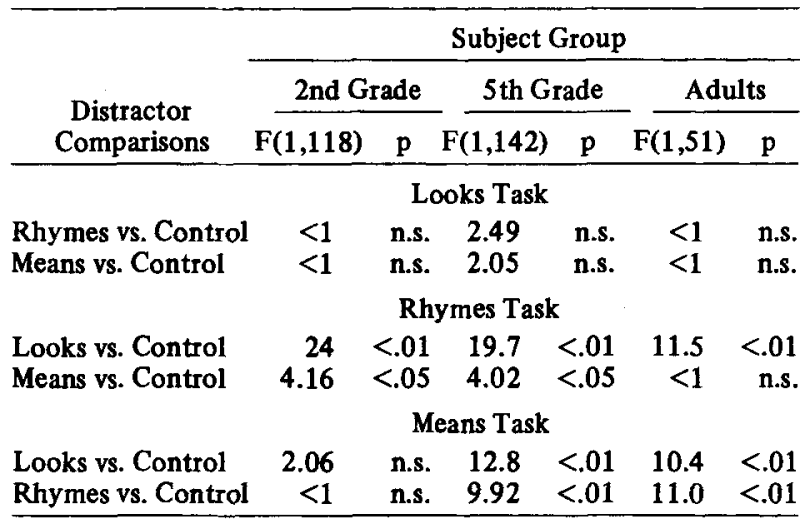

which presents the RTs for the three grades for each task-distractor cell.

The interaction between distractor and grade was tested in the same fashion as overall distractor effectiveness. The analysis of variance indicated a nearly significant Grade by Distractor effect $[F(1,313)=$. $2.4, p<.10$ ]. This interaction, shown in Figure 2, takes the form of diminishing effects of distractor type with age.

Distractor effects on each task at the three grade levels are given in Table 3. It is evident that neither type of distractor disturbed the looks task. On the other hand, both types of distractors affected the means task for fifth graders and adults, though not for the youngest group. A more complex pattern of results appeared in the rhymes task, with some significant effects at each grade level. ${ }^{5}$

Table 4 presents the proportion of errors at each grade level for each of the nine task-distractor treatments, and also for each grouping condition. The error rates were quite low. As can be seen, the error data follow the same overall pattern as the RT data. There was a significant product-moment correlation $(r=+.80)$ between number of errors over subjects in a given Grade by Task by Distractor cell, and the corresponding average RT for correct responses, which suggests that no speed-accuracy tradeoff occurred.

\section{DISCUSSION}

The study reported here was devised to examine the development of ability to extract information from words. Even the youngest subjects were able to perform the task of selecting a given feature of a word reasonably well. Older subjects responded faster and more accurately than younger subjects on all combinations of tasks, distractors, and grouping conditions, resulting in the large, reliable main effect of grade. This result was expected on the basis of development in reading skills, as well as in speed of simple RT and ability to make similarity judgments.

The relative difficulty of the three tasks decreased with age. Choosing a word that looks like another was easy (relative to the other tasks) at each age. Judging orthographic similarity (at least within the range of words tested here) is a skill that develops early. It is this ability that is tapped when young readers are introduced to rhyming words that share a spelling pattern. The rhymes task was significantly more difficult than the looks task at all ages. Finding a word that rhymes when there is not a common spelling pattern to rely on is a difficult task (relative to the looks-alike task) at any stage of reading ability. It requires decoding to sound and, to some extent, ignoring the dissimilar spelling. The task is made significantly harder when one choice is a word with similar spelling (looks distractor) even for adults. Choosing the word with similar meaning gets easier with age. For adults, the means task is no more difficult than the rhymes task. For second graders, it is significantly more difficult. The words were selected for their likely familiarity to elementary age children. Even so, second graders had trouble finding a synonym. The problem is most likely due to the lack of sentence context-a source of information less necessary to adults. The means task was not too advanced for the youngest subjects-they performed the task with only a moderate error rate. In sum, the Grade by Task interaction suggests developmental progress (primarily between second and fifth grade) in facility of extracting phonetic and semantic information from words.

We expected, overall, that skilled readers performing our tasks would be less distracted by irrelevant

Table 4

Proportion of Errors Made by Subjects in Various Conditions

\begin{tabular}{|c|c|c|c|c|}
\hline \multirow[b]{2}{*}{ Task } & \multirow[b]{2}{*}{ Distractor } & \multicolumn{3}{|c|}{ Grade } \\
\hline & & 2 & 5 & Adult \\
\hline \multicolumn{5}{|c|}{ A. The Nine Task-Distractor Conditions } \\
\hline Looks & $\begin{array}{l}\text { Control } \\
\text { Rhymes } \\
\text { Means }\end{array}$ & $\begin{array}{l}.019 \\
.083 \\
.057\end{array}$ & $\begin{array}{l}.020 \\
.068 \\
.036\end{array}$ & $\begin{array}{l}.015 \\
.100 \\
.000\end{array}$ \\
\hline Rhymes & $\begin{array}{l}\text { Control } \\
\text { Looks } \\
\text { Means }\end{array}$ & $\begin{array}{l}.029 \\
.166 \\
.055\end{array}$ & $\begin{array}{l}.024 \\
.166 \\
.044\end{array}$ & $\begin{array}{l}.030 \\
.115 \\
.045\end{array}$ \\
\hline Means & $\begin{array}{l}\text { Control } \\
\text { Looks } \\
\text { Rhymes }\end{array}$ & $\begin{array}{l}.093 \\
.138 \\
.176\end{array}$ & $\begin{array}{l}.026 \\
.058 \\
.074\end{array}$ & $\begin{array}{l}.030 \\
.035 \\
.080\end{array}$ \\
\hline \multicolumn{5}{|c|}{ B. Grouping of Trials } \\
\hline & $\begin{array}{l}\text { Blocked } \\
\text { Unblocked }\end{array}$ & $\begin{array}{l}.081 \\
.102\end{array}$ & $\begin{array}{l}.039 \\
.076\end{array}$ & $\begin{array}{l}.057 \\
.043\end{array}$ \\
\hline
\end{tabular}


similarities (feature distractors) than would less skilled readers. The Grade by Distractor interaction that we expected was observed, but was only marginally significant across three grades and was not significant between second and fifth grades. Our results thus do not provide strong evidence for a developmental change toward filtering out irrelevant information. The presence of task-irrelevant confusable information continued to exert significant effects even on experienced readers.

The effects of particular distractors varied from task to task. On the looks task, the feature distractors had no significant effects on subjects at any age.

The looks distractors made the rhymes task more difficult at each age. In the present experimental situation, where it would have been more economical to ignore graphic similarities, young readers had difficulty doing so. For many words, the tendency to respond as if similar spelling implies similar pronunciation is a reasonable strategy and is encouraged in early reading training. This tendency is markedly reduced with age, although it does not disappear. The means distractor on the rhymes task slowed RT for second and fifth graders but not for adults, a finding consonant with the view that there is an early tendency to extract meaning from words spontaneously (see also Rosinski, Golinkoff, \& Kukish, 1975). Older subjects were better able to ignore irrelevant information, even meaning.

On the means task for the second graders, feature distractors were not more disruptive than control distractors. However, they were for fifth graders and adults. It is possible that the difficulty of the task (as indexed by long and variable RTs) obscured any feature distractor effects for second graders.

As pointed out in the introduction, the rhymes distractor data bear on the phonemic recoding view of reading acquisition-that development in reading skill involves a gradual shift from dependence on the sound of printed words to direct access to meaning from print. This view would predict that the effect of the rhymes distractor on the means task should decrease with age as reliance on sound decreases. This did not occur. Our data support Barron and Baron's (1977) recent finding on this point.

Blocked and unsystematic grouping of the three tasks was manipulated to test development of flexibility of attentional strategies. Contrary to our hypothesis, it was found that a blocked presentation of each task facilitated performance at all ages. While there was a tendency for second-grade subjects to benefit more from blocking than older subjects, it was nonsignificant, and very small. The youngest children's RTs were $27 \%$ faster on blocked trials than on unsystematic trials; the advantage of blocking for both groups of older subjects was $24 \%$. Thus, the development in flexibility which has emerged in some previous studies did not obtain in the present experiment. Flexibility, per se, may not be a general skill but one which is specific to particular tasks. Reading requires skills that are normally directed toward deriving meaning. The absence of a developmental trend in the present study suggests that, on the whole, skilled readers have not needed or practiced the type of flexibility sought in this study. The purpose of this study was to explore developmental changes in ability to work with specific feature classes when the information is printed words. A recurrent question in the study of reading acquisition is the extent to which reading is "parasitical on speech" - merely a step tacked on after a child has control of language by virtue of understanding and producing speech. The three tasks used in this study could be presented aurally, with minor modifications, and be compared with the present results. This would give an interesting perspective on the differences between listening and reading. The looks task would certainly increase in difficulty, the rhymes task should decrease. Rhymes and looks distractor effects should switch places in their relative interference effects.

As argued by Golinkoff (1975-76), it is of practical import that we determine the differences between good and poor readers of the same age. The present task-distractor experimental paradigm should provide useful diagnostic information. There are several advantages to this paradigm. The measures are sensitive across a wide range of ages as evidenced by the significant task and distractor effects for both second graders and adults. The instructions are readily understandable by young children. The inclusion of control distractors makes the effects of looks, rhymes, and means distractors easily interpretable.

The major conclusion of this study is that children as young as second grade are able to attend selectively (upon demand) to varied kinds of information specified by printed words. They adapt their attending, working with graphic, phonetic, or semantic information when it is useful for the particular task at hand. There is, overall, an increasing (but not complete) ability to ignore task-irrelevant information. The results do not support the notion that there is a developmental shift from dependence on the sound of printed words to direct access to meaning from print.

\section{REFERENCE NOTE}

1. Golden, C. The child's perception and awareness of the phonemes in words. Paper presented at 47th Annual Meeting of Eastern Psychological Association in New York City, April 1976.

\section{REFERENCES}

ANDERson, I. H. Studies in the eye movements of good and poor readers. Psychological Monographs, 1973, 48(Whole No. 215), 25-35. 
BARRoN, R., \& BARON, J. How children get meaning from printed words. Child Development, 1977, 48, 587-594.

BoG ARTZ, R. S. On the meaning of statistical interactions. Journal of Experimental Child Psychology, 1976, 22, 178-183.

Clifton, C., \& Bogartz, R. Selective attention during dichotic listening by preschool children. Journal of Experimental Child Psychology, 1968, 6, 483-491.

DoyLE. A.-B. Listening to distraction: A developmental study of selective attention. Journal of Experimental Child Psychology, 1973, 15, 100-115.

Gibson, E. J. Perceptual learning and the theory of word perception. Cognitive Psychology, 1971, 2, 351-368.

Gibson, E. J.. \& YonAs, A. A developmental study of visual search behavior. Perception \& Psychophysics, 1966, 1, 169-171.

GolinkofF, R. A comparison of reading comprehension processes in good and poor comprehenders. Reading Research Quarterly, 1975.76, 11, 623-659.

Maccoby, E. E., \& Konrad, K. W. Age trends in selective listening. Journal of Experimental Child Psychology, 1966, 3, 113-122.

MACCOBY, E. E., \& KonRAD, K. W. The effect of preparatory set on selective listening: Developmental trends. Monograph of the Society for Research in Child Development, 1967.

Pick, A. D. . Christy, M. D., \& Frankel, G. W. A developmental study of visual selective attention. Journal of Experimental Child Psychology, 1972, 14, 165-176.

Pick, A. D., \& Frankel, G. W. A study of strategies of visual attention in children. Developmental Psychology, 1973, 9 348-357.

Rosinski, R. R., Golinkoff, R. M., \& Kukish, K. S. Automatic semantic processing in a picture-word interference task. Child Development, 1975, 46, 247-253.

Smith, L. B., Kemler, D. G., \& Aronfreed, J. Developmental trends in voluntary selective attention: Developmental effects of source distinctness. Journal of Experimental Child Psychology, $1975,20,352-362$.

Strutt, G. F., Anderson, D. R., \& Well, A. D. A developmental study of the effects of irrelevant information of speeded classification. Journal of Experimental Child Psychology, 1975, 20. 127-135.

THORndike, E. L., \& LORGE, I. The teacher's word book of 30,000 words. New York: Bureau of Publications, Teacher's College, Columbia University. 1944.

WINER, B. J. Statistical principles of experimental design. New York: McGraw-Hill, 1971.

\section{NOTES}

1. Data from nine second graders were dropped from statistical analyses because they made more than $25 \%$ errors or because they did not complete the entire 90 trials. The school children were tested in January. Reading instruction used in the school was the Distar program by S. Engelmann and colleagues.

2. The entire list of $\mathbf{2 7 0}$ words is available to interested readers.

3. As pointed out by Bogartz. (1976), a proportional change model may be an appropriate model for determining effects interacting with age. Proportional changes in time to perform the three tasks in each of the three grades, based on cell means, agree with the results of the paired comparisons. The difference between the looks and rhymes task and between the looks and means task decreases mainly between second and fifth grade. The difference between the rhymes and means tasks decreases far less with age.

4. The confounding of distractor with task was minimized in this comparison by balancing the occurrence of the three tasks between the distractors compared, as follows: the control distractor RTs collapsed over grade, grouping, and task and were compared with the other three distractors combined, also collapsed over grade, grouping, and task. The test may be described as control distractor vs. others, or as $2 D_{c}-\left(D_{1}+D_{r}+D_{m}\right)$, where $D_{c}$ represents the control distractor at all three task levels (three cells of the 3 by 4 design), $D_{1}$ is the looks distractor on the rhymes and means tasks (two cells), $D_{r}$ is the rhymes distractor on the looks and means tasks (two cells), and $D_{m}$ is the means distractor on the looks and rhymes tasks (two cells). Thus, each task was included once (multiplied by 2 ) on the control distractor side of the comparison, and twice on the others side. Since the tasks were thus evenly represented for control and others, the confounding of distractor with task was essentially eliminated.

5. Proportional changes of feature vs. control distractors at each age, based on cell means, indicates that feature distractors have little effect on the looks task at all ages; on the rhymes task, feature distractor effects decrease with age; on the means task, feature distractor effects increase with age.

(Received for publication August 11, 1978; revision accepted November 7,1978 .) 